Delft University of Technology

\title{
UWB orthogonal pulse design using Sturm-Liouville boundary value problem
}

Amini, Arash; Mohajerin Esfahani, Peyman; Ghavami, Mohammad; Marvasti, Farokh

DOI

10.1016/j.sigpro.2019.02.008

Publication date

2019

Document Version

Final published version

Published in

Signal Processing

\section{Citation (APA)}

Amini, A., Mohajerin Esfahani, P., Ghavami, M., \& Marvasti, F. (2019). UWB orthogonal pulse design using Sturm-Liouville boundary value problem. Signal Processing, 159, 147-158.

https://doi.org/10.1016/j.sigpro.2019.02.008

\section{Important note}

To cite this publication, please use the final published version (if applicable).

Please check the document version above.

\section{Copyright}

Other than for strictly personal use, it is not permitted to download, forward or distribute the text or part of it, without the consent of the author(s) and/or copyright holder(s), unless the work is under an open content license such as Creative Commons.

\section{Takedown policy}

Please contact us and provide details if you believe this document breaches copyrights.

We will remove access to the work immediately and investigate your claim. 
Green Open Access added to TU Delft Institutional Repository

'You share, we take care!' - Taverne project

https://www.openaccess.nl/en/you-share-we-take-care

Otherwise as indicated in the copyright section: the publisher is the copyright holder of this work and the author uses the Dutch legislation to make this work public. 


\title{
UWB orthogonal pulse design using Sturm-Liouville boundary value problem
}

\author{
Arash Amini ${ }^{a}$, Peyman Mohajerin Esfahani ${ }^{\mathrm{b}}$, Mohammad Ghavami ${ }^{\mathrm{c}, *}$, Farokh Marvasti ${ }^{\mathrm{a}}$ \\ a Sharif University of Technology, Advanced Communication Research Institute, Tehran, Iran \\ ${ }^{\mathrm{b}}$ Delft University of Technology, Delft Center for Systems and Control, The Netherlands \\ ${ }^{c}$ London South Bank University, School of Engineering, London, UK
}

\section{A R T I C L E I N F O}

\section{Article history:}

Received 22 October 2018

Revised 25 January 2019

Accepted 6 February 2019

Available online 8 February 2019

\section{Keywords:}

Orthogonal UWB pulses

Sturm-Liouville theory

Spectral mask

\begin{abstract}
A B S T R A C T
The problem of designing UWB pulses which meet specific spectrum requirements is usually treated by filtering common pulses such as Gaussian doublets, modified Hermite polynomials and wavelets. When there is the need to have a number of orthogonal pulses (e.g., in a multiuser scenario), a naive approach is to filter all the members of an orthogonal set, which is likely to destroy their orthogonality property. In this paper, we study the design of a set of pulses that simultaneously satisfy the orthogonality property and spectrum requirements. Our design is based on the eigenfunctions of Sturm-Liouville boundary value problems. Indeed, we introduce Sturm-Liouville differential equations for which the eigenfunctions meet the FCC mask constraints. Computer simulation results show that all such waveforms occupy almost 55\% of the allowed spectrum (utilization efficiency). A comparison of the proposed method with some conventional techniques of orthogonal UWB pulse generation will demonstrate the advantages of the new proposal.
\end{abstract}

(c) 2019 Elsevier B.V. All rights reserved.

\section{Introduction}

Ultra Wideband (UWB) technology is one of the well-known solutions for short range and low energy transmission of high data rate signals. Historically, its traditional application used to be in radar [1] while new applications consist of wireless communication [2], transmission of multimedia signals $[3,4]$ and sensor networks $[5,6]$.

For many years, single pulse generation has been the traditional and fundamental approach for creating UWB waveforms. By adjusting the pulse parameters, the characteristics of the energy within the frequency spectrum may be defined based on some desired design criteria. The parameters such as the pulse duration, bandwidth and the energy should be carefully considered. The location where the generated energy should center is also important. Pulse shape determines the characteristics of how the energy occupies the frequency domain. Since the highest spectral efficiency is one of the most important objectives for UWB communications, as much bandwidth as is practical should be used to take advantage of the capacity made available with UWB technology [7].

Generally, single-pulse architectures offer relatively simple radio designs, but provide little flexibility where spectrum

\footnotetext{
* Corresponding author.

E-mail address: ghavamim@lsbu.ac.uk (M. Ghavami).
}

management is an objective. Examples of scenarios where managing the spectrum might be desirable are matching different regulatory requirements in different international regions, dynamically sensing interfering technologies and suspending usage of contending frequencies. Another area where managing the spectrum might be desirable is in performance management. Increasing the performance of existing designs may require a complete redesign, forgoing backward compatibility with earlier implementations [2].

UWB transmission can be carried out by sending nanosecond pulses which represent a single or a number of bits. The most adopted waveforms are Gaussian monocycle and doublet [8,9]; however, other waveforms such as Hermites and spheroidal prolates are also considered [2,10,11]. Unlike the Gaussian pulses, the latter classes include a number of pairwise orthogonal pulses which can be used either for transmission of multi-bit symbols via orthogonal shapes or single-bit waveforms of a multi-user communication system. Hence, it is beneficial to use a number of orthogonal pulses rather than a single waveform.

Since UWB pulses occupy a wide range of the available spectrum, it is likely that the pulses cause interference in the preassigned frequency bands. In order to alleviate this effect, a number of frequency masks, such as the one by FCC [12], are regulated. These masks consist of disjoint frequency intervals for which the transmitted pulses should be kept below a constant level. In order to meet the mask requirements, usually the employed 
waveforms are passed through pulse shaping filters [13,14]. If a set of orthogonal waveforms are subject to the filtering process, the outputs are likely not to be orthogonal. Hence, for having orthogonal pulses that meet the mask requirements, instead of shaping the well-known orthogonal sets, one should design new pulses such as the ones introduced in [13]. Since the limiting levels of the mask in the adjacent frequency intervals discontinuously change and the transmitted pulses are essentially continuous, the allowable spectrum could not be fully employed. To evaluate the efficiency of a waveform with respect to a frequency mask, it is common to report the used fraction of the allowable spectrum as the spectrum utilization efficiency.

It is shown that the common pulses introduced for UWB transmission follow a second order differential equation known as Sturm-Liouville boundary value problem [15] which posses orthogonal eigenfunctions with respect to a given weight-function. In this paper, by focusing on the classes of Sturm-Liouville functions, we will introduce new orthogonal sets of pulses which meet the FCC mask requirements. This goal is achieved by forcing a tunable null in the frequency spectrum of the pulses which is also useful for avoiding interference in a narrow-band frequency interval. Although we consider the FCC mask, the pulse generation algorithm can be adapted for other frequency requirements. An interesting property of the obtained pulses, which we observe in simulation results, is that the utilization efficiency of different pulses are almost the same (about 55\%) which is not the case for the pulses introduced in [13]. This results in a fair distribution of the waveforms among the users for a multi-access scenario.

The rest of the paper is organized as follows: In Section 2 we review some preliminaries about pulse requirements and SturmLiouville theory. Our approach in designing pulses that meet the FCC requirements is explained in Section 3. We introduce a set of Sturm-Liouville boundary value problems in Section 4 that include potential UWB waveforms as their eigenfunctions. In Section 5, we validate the theoretical results by simulations for an instance of the proposed sets. Section 6 compares the proposed method with some other approaches and finally Section 7 presents the conclusion.

\section{Preliminaries}

Now we briefly explain the desired properties of UWB pulses and review the basics of Sturm-Liouville theory.

\subsection{UWB pulse requirements}

The following summarizes the criteria that a single or set of UWB pulses should satisfy:

1. Finite energy: The most elementary pulse requirement which is not restricted to UWB communications is that the energy of the pulse should be finite. Although it is trivial to have such pulses, when differential equations are the design tools, this requirement should be checked. This condition also implies the finite effective duration in time; i.e., the time period that contains $99 \%$ of the pulse energy is bounded.

2. Zero DC: The DC or low frequency components of waveforms increases the dynamic range of the amplifiers and shifts the operating mode of the devices towards the nonlinear regime. Besides, in some UWB scenarios, the transmitting antenna differentiates the waveform, which automatically removes the DC component. Hence, the UWB pulses should contain no DC component.

3. Orthogonality: As mentioned, we are looking for a set of orthogonal pulses. Thus, pulses should be orthogonal. This condition is the main motivation behind the choice of Sturm-Liouville functions as UWB pulses.
4. Spectrum mask: Constraints on the spectrum of the transmitting pulse should also be considered. That is, the spectral content of the pulses should be below a given level imposed by a regulatory mask. This is the main challenge in UWB pulse design.

5. Utilization efficiency: The last requirement is to maximize the employed fraction of the allowed spectrum measured as utilization efficiency factor. To measure the efficiency a normalized effective signal power (NESP) is defined. For a pulse $y(t)$ with upper bound mask $\mathrm{M}(\omega)$, NESP is defined as [13]

$\operatorname{NESP}(y)=\frac{\int|\hat{y}(\omega)|^{2} \mathrm{~d} \omega}{\int \mathrm{M}(\omega) \mathrm{d} \omega}$,

where $\hat{y}(\omega)$ represents the Fourier transform of $y(t)$. Although higher factors are desirable in a single-user communication protocol, it is preferable that different pulses in a multi-user UWB system have similar utilization efficiencies (fair distribution).

\subsection{Sturm-Liouville theory}

In the field of ordinary differential equations (ODE), a subclass of boundary value problems referred to as Sturm-Liouville equations, are known to be involved in many physical phenomena such as vibration. The ODE structure

$\frac{\mathrm{d}}{\mathrm{d} t}[p(t) \dot{y}(t)]+[q(t)+\lambda s(t)] y(t)=0$

accompanied with boundary conditions at $t_{l}, t_{u}$ of the form

$\left\{\begin{array}{l}c_{1} y\left(t_{l}\right)+c_{2} \dot{y}\left(t_{l}\right)=0, \\ c_{3} y\left(t_{u}\right)+c_{4} \dot{y}\left(t_{u}\right)=0,\end{array}\right.$

is called a Sturm-Liouville boundary value problem. We reserve the notations $\dot{y}$ and $\ddot{y}$ to represent the first and second order derivatives of the function $y$. The functions $p(\cdot), q(\cdot), s(\cdot)$ in (2) are continuous with $p(\cdot)$ and $s(\cdot)$ differentiable and positive-valued within $\left[t_{l} t_{u}\right]$. If $y(\cdot)$ is a nontrivial solution of (2) for some $\lambda$ and satisfies (3), it is called an eigenfunction of the problem, and the corresponding $\lambda$ is known as an eigenvalue. Eigenvalues form a monotonically increasing sequence of real numbers with countable infinite cardinality [16]:

$\lambda_{0}<\lambda_{1}<\cdots \rightarrow \infty$.

One can check that if $\left(y_{n}(\cdot), \lambda_{n}\right)$ and $\left(y_{m}(\cdot), \lambda_{m}\right)$ are two eigenpairs of the problem, we have that

$$
\begin{aligned}
& \left(\lambda_{n}-\lambda_{m}\right) \int_{t_{1}}^{t_{2}} y_{n}(t) y_{m}(t) s(t) \mathrm{d} t \\
& \quad=\left.p(t)\left(\dot{y}_{n}(t) y_{m}(t)-\dot{y}_{m}(t) y_{n}(t)\right)\right|_{t_{1}} ^{t_{2}}
\end{aligned}
$$

Setting $t_{1}=t_{l}$ and $t_{2}=t_{u}$ in (5) and using (3) results:

$\forall m \neq n: \quad \int_{t_{l}}^{t_{u}} y_{n}(t) y_{m}(t) s(t) \mathrm{d} t=0$,

which shows the orthogonality of eigenfunctions with respect to the weight function $s(t)$ over the interval determined by the boundary conditions.

Besides the orthogonality in the interval $\left[t_{l}, t_{u}\right]$, we use (5) to show that under some conditions, we can select a subset of eigenfunctions with arbitrarily small pairwise inner products (almost orthogonality) over the interval $\left[t_{u}, \infty\right)$. Since there are no boundary conditions at $t=\infty$, we need to study the asymptotic behavior of the eigenfunctions to establish the latter property. 


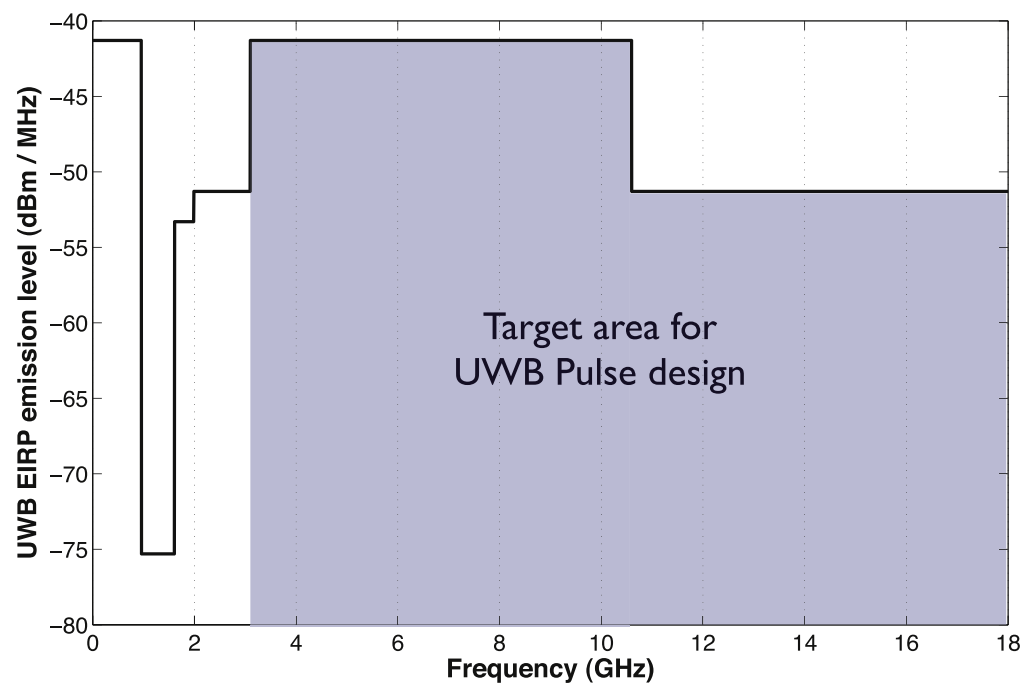

Fig. 1. The FCC mask for indoor UWB communications and the target area for pulse design.

\section{Design approach}

In this section, we establish the link between the solutions of a special type of Sturm-Liouville boundary value problem and the waveforms suitable for UWB communications.

Since the restrictions imposed by UWB regulatory bodies are presented in frequency, we focus on designing Sturm-Liouville differential equations in the frequency domain for which the eigenfunctions meet the UWB requirements. To distinguish between time and frequency, we denote the pulse in the frequency-domain (Fourier transform) by $\hat{y}(\omega)$ while $y(t)$ represents the pulse in the time-domain. Due to the Parseval's theorem, the inner product of the pulses are identical in both domains. In particular, the pulses are orthogonal in one domain if and only if they are orthogonal in the other one.

For $100 \%$ utilization of the permitted spectra, the waveforms should have the same frequency content as the mask while maintaining the orthogonality property. Fig. 1 depicts a particular mask known as the FCC mask. We observe that the level restrictions vary discontinuously at the borders of non-overlapping adjacent frequency intervals. Thus, a waveform that achieves $100 \%$ utilization $^{1}$ of this mask should be unstable in time-domain. In other words, it is not absolutely summable which indicates the long time-duration of the pulse which is not feasible in practice, especially when the transmission rate is high and the receiver suffers from timing offsets.

An alternative approach is to initially ignore the spectrum requirements and select an orthogonal set of pulses which have desirable time-duration. Then, it is possible to force the FCC mask requirements by attenuating the pulses. The proper attenuation factor depends only on the maximum frequency content of the pulse relative to the mask. This suggests that the whole spectrum should be suppressed to avoid a small part surpassing the limits. The consequence is that we obtain pulses with disappointing utilization efficiency. One may think of applying various attenuation factors in different frequency intervals. Although it usually results in considerable improvements in the utilization efficiency, it destroys the orthogonality of the pulses. Furthermore, the variable attenuation factor in different intervals may cause discontinuity at the borders which again translates into time instability of pulses.

\footnotetext{
${ }^{1}$ Here, the mask is considered up to an upper bound over the frequency axis; otherwise, the mask-matching waveform would have infinite energy.
}

Here, we try to employ the variable attenuation technique by avoiding its side effects. The UWB FCC mask with six distinguished sections, each with a certain level of power spectrum, suggests that we partition the frequency axis into six consecutive intervals (see Fig. 1). We represent the borders of these intervals by $\left\{\omega_{i}\right\}_{i=0}^{6}$, where $\omega_{0}=0$ and $\omega_{6}=\infty$. Let us first assume the existence of a set of pulses $\left\{\hat{y}_{n}(\omega)\right\}_{n}$ that are pairwise orthogonal over each of the frequency sub-intervals $\left[\omega_{i}, \omega_{i+1}\right]$, while each pulse vanishes at the borders of the sub-intervals. In other words,

$\int_{\omega_{i}}^{\omega_{i+1}} \hat{y}_{n}(\omega) \overline{\hat{y}_{m}(\omega)} \mathrm{d} \omega=0, \quad 0 \leq i \leq 5$,

for all $m \neq n$ and $\hat{y}_{n}\left(\omega_{i}\right)=0,0 \leq i \leq 6$. Due to the vanishing property of the pulse spectra at the borders, the variable attenuation technique does not cause any discontinuity in the spectra. In addition, because of the piecewise orthogonality of the waveforms, they remain orthogonal after applying the variable attenuation technique as long as the attenuation factors are fixed within sub-intervals. Thus, the main challenge is to find a set of pulses that satisfy the aforementioned constraints.

For design simplicity, similar to $\mathrm{Wu}$ et al. [13], we neglect the permitted spectra below $3.1 \mathrm{GHz}$. In other words, we try to find pairwise orthogonal pulses that have nonzero frequency content only for $\frac{\omega}{2 \pi}>3.1 \mathrm{GHz}$ and are orthogonal in both intervals: 3.110.6 GHz and $10.6 \mathrm{GHz}-\infty$. We also impose the constraint that the waveforms vanish at $\frac{\omega}{2 \pi}=3.1 \mathrm{GHz}$ and $\frac{\omega}{2 \pi}=10.6 \mathrm{GHz}$. It should be noted that the practical way of applying piecewise constant attenuation in the frequency is via time domain filtering. In each frequency interval, we should apply an ideal bandpass filter for which the passband amplitude is determined by the desired attenuation factor. Since practical filters have nonzero transition band (nonideal), the filtering process causes distortion in the adjacent bands and consequently, it affects the overall orthogonality. Another advantage of imposing notches at $\frac{\omega}{2 \pi}=3.1 \mathrm{GHz}$ and $\frac{\omega}{2 \pi}=10.6 \mathrm{GHz}$ is to minimize this effect by decreasing the energy level in the transition bands.

\section{Proposed pulses}

In this section, we propose a class of Sturm-Liouville differential equations in the frequency-domain for which the eigenfunctions have finite energy and are pairwise orthogonal over the frequency band $\left[\omega_{l}, \omega_{u}\right]$. We establish the existence of a proper subset of the eigenfunctions that satisfy the almost orthogonality 
property (see Definition 1) over the frequency band $\left[\omega_{u}, \infty\right)$. As a special case, we can set $\frac{\omega_{l}}{2 \pi}=3.1 \mathrm{GHz}$ and $\frac{\omega_{u}}{2 \pi}=10.6 \mathrm{GHz}$ to adapt the waveforms to the requirements of the FCC mask. However, the procedure is transparent to the choice of $\omega_{l}, \omega_{u}$ and can be applied to other masks. In all of our simulations, we observe that the eigenfunctions are orthogonal rather than almost orthogonal over the interval $\left[\omega_{u}, \infty\right)$, and there is no need to wisely select a subset of them. Another advantage of the proposed pulses is that they satisfy differential equations in both time and frequency domains.

\subsection{The proposed set of differential equations}

We consider differential equations of the form (2), where the functions $p, q$ and $s$ are polynomials. The primary benefit of this choice is that the differential equation in the frequency-domain corresponds to another differential equation in the time-domain; further details are provided in Section 4.4. The other benefit, which is stated in Theorem 1, is the decay property of the eigenfunctions. We recall that the differential equations are considered in the frequency-domain; hence, the references to (2) and (3) implicitly include replacement of $t$ (time index) with $\omega$ (frequency index).

Theorem 1. Let $\left\{\hat{y}_{n}(\omega)\right\}_{n}$ and $\left\{\lambda_{n}\right\}_{n}$ be the eigenfunctions and eigenvalues of the problem (2), respectively, with the boundary conditions at $0 \leq \omega_{l}<\omega_{u}$ similar to (3), where

$p(\omega)=\sum_{i=0}^{k} a_{i} \omega^{i}, \quad q(\omega)=\sum_{i=0}^{k-2} b_{i} \omega^{i}, \quad s(\omega) \equiv 1$

such that $k \geq 4, a_{i}, b_{i} \geq 0, a_{k}, a_{k-1} \neq 0$ and

$\frac{b_{k-2}}{a_{k}}>\left(\frac{k-1}{2}\right)^{2}$,

$\frac{b_{k-3}}{a_{k-1}}+\frac{b_{k-2}}{a_{k}}<\left(\frac{k-1}{2}\right)^{2}+\frac{k^{2}-4 k+3}{4}$.

Then,

1. $\left\{\hat{y}_{n}\right\}_{n}$ are pairwise orthogonal over $\left[\omega_{l}, \omega_{u}\right]$,

2. $\omega^{\frac{k-1}{2}} \hat{y}_{n}(\omega)$ and $\omega^{\frac{k+1}{2}} \dot{\hat{y}}_{n}(\omega)$ are bounded,

3. $\left\{\hat{y}_{n}\right\}_{n}$ have finite energy,

4. There exist $\left\{\bar{\theta}_{n}\right\}_{n}$ and a constant $c$ such that for all $n$ and $m$

$$
\left(\lambda_{n}-\lambda_{m}\right) \int_{\omega_{u}}^{\infty} \hat{y}_{n}(\omega) \hat{y}_{m}(\omega) \mathrm{d} \omega=c M_{\hat{y}_{n}} M_{\hat{y}_{m}} \sin \left(\bar{\theta}_{n}-\bar{\theta}_{m}\right),
$$

where

$$
M_{\hat{y}_{n}}=\limsup _{\omega \rightarrow \infty} \omega^{\frac{k-1}{2}} \hat{y}_{n}(\omega) \text {. }
$$

To facilitate reading of the paper, the proof of Theorem 1 is postponed to Appendix A.

Claim 4 of Theorem 1 focuses on the inner product of the eigenfunctions. The ideal result would be to demonstrate orthogonality. Instead, the weaker result of (11) can be applied to demonstrate almost orthogonality. Due to the structure (3) of boundary conditions, any multiple of an eigenfunction is again an eigenfunction. Thus, their inner products are meaningful only when normalized. The quantity

$\frac{\int_{\omega_{u}}^{\infty} \hat{y}_{n}(\omega) \hat{y}_{m}(\omega) \mathrm{d} \omega}{M_{\hat{y}_{n}} M_{\hat{y}_{m}}}$

shows one possible choice. This suggests that by decreasing $\sin \left(\bar{\theta}_{n}-\bar{\theta}_{m}\right)$ we can decrease the normalized inner products.

Definition 1. The set of functions $\left\{\hat{y}_{n}(\omega)\right\}_{n}$ is called almost orthogonal if for any positive integer $N$ and any $\epsilon>0$, one can choose a subset $\hat{y}_{i_{1}}, \ldots, \hat{y}_{i_{N}}(\omega)$ such that

$\forall 1 \leq j, k \leq N, j \neq k, \quad\left|\frac{\int \hat{y}_{i_{j}}(\omega) \hat{y}_{i_{k}}(\omega) \mathrm{d} \omega}{M_{\hat{y}_{i_{j}}} M_{\hat{y}_{i_{k}}}}\right| \leq \epsilon$.

We show that the set of eigenfunctions introduced in Theorem 1 is almost orthogonal. Since $\lambda_{i} \rightarrow \infty$, it is possible to find a subset $\mathcal{S}=\left\{s_{1}, s_{2}, \ldots\right\} \subset \mathbb{N}^{+}$such that for all $s_{j} \neq s_{k}$ we have that $\left|\lambda_{s_{k}}-\lambda_{s_{j}}\right| \geq c$, where $c$ is the same constant as in (11). Now, for given $N \in \mathbb{N}^{+}$and $\epsilon>0$, let $d$ be the least integer greater than $\frac{2 \pi}{\epsilon}$ and define $\overline{\mathcal{S}}=\left\{s_{1}, \ldots, s_{d(N-1)+1}\right\}$. Consider the partitioning of the interval $[0,2 \pi)$ into $d$ subintervals of length $\frac{2 \pi}{d}$ (bins). According to the Pigeonhole principle, any set of $d(N-1)+1$ numbers in the range $[0,2 \pi)$ consists of an $N$-element subset all located in the same bin. Thus, there exist $i_{1}, \ldots, i_{N} \in \overline{\mathcal{S}}$ such that $\bar{\theta}_{i_{1}}, \ldots, \bar{\theta}_{i_{N}}$ are all in the same bin. Hence, for $j \neq k$ in $\{1, \ldots, N\}$, by applying (11) we conclude that

$$
\begin{aligned}
\left|\frac{\int_{\omega_{u}}^{\infty} \hat{y}_{i_{j}}(\omega) \hat{y}_{i_{k}}(\omega) \mathrm{d} \omega}{M_{\hat{y}_{i_{j}}} M_{\hat{y}_{i_{k}}}}\right| & =\left|\frac{c}{\lambda_{i_{j}}-\lambda_{i_{k}}}\right|\left|\sin \left(\bar{\theta}_{i_{j}}-\bar{\theta}_{i_{k}}\right)\right| \\
& \leq\left|\sin \left(\bar{\theta}_{i_{j}}-\bar{\theta}_{i_{k}}\right)\right| \leq \sin \left(\frac{2 \pi}{d}\right)<\frac{2 \pi}{d} \leq \epsilon .
\end{aligned}
$$

This establishes the existence of a suitable subset of eigenfunctions such as $\left\{\hat{y}_{i_{k}}(\omega)\right\}_{k=1}^{N}$ that are pairwise orthogonal over $\left[\omega_{l}, \omega_{u}\right]$ and almost orthogonal over $\left[\omega_{u}, \infty\right)$. It is worth mentioning that in all of our simulations we observe that $M_{\hat{y}_{n}}=0$, which in turn indicates that $\hat{y}_{n} \mathrm{~s}$ are exactly orthogonal (rather than almost orthogonal) up to the numerical precision. This leads us to the following conjecture.

Conjecture 1. If $\left\{\hat{y}_{n}\right\}_{n}$ denote the eigenfunctions of the SturmLiouville problem specified in Theorem 1, for positive integers $n \neq m$ we have that

$\int_{\omega_{u}}^{\infty} \hat{y}_{n}(\omega) \hat{y}_{m}(\omega) \mathrm{d} \omega=0$

\subsection{Boundary conditions}

As argued in Section 3, due to non-ideality of analog filters, it is preferable to decrease the energy in the transition bands of the filters. To this end, we tune $\omega_{l}$ and $\omega_{u}$ of Theorem 1 to match the transition bands of filters and anchor them to zero. That is, we set the boundary conditions in the form of

$\hat{y}_{n}\left(\omega_{l}\right)=\hat{y}_{n}\left(\omega_{u}\right)=0$,

where $\omega_{l}$ and $\omega_{u}$ depend on the spectrum requirements. Note that (15) is a special case of (3) obtained for $c_{1}=c_{3}=1$ and $c_{2}=c_{4}=$ 0 . For the FCC mask of Fig. 1, we choose $\frac{\omega_{l}}{2 \pi}=3.1 \mathrm{GHz}$ and $\frac{\omega_{u}}{2 \pi}=$ $10.6 \mathrm{GHz}$ which are the main discontinuities of the spectrum. We implicitly assume that the band $\left[0, \omega_{l}\right]$ will be eventually discarded by means of filtering. The key message is that tunability of $\omega_{l}$ and $\omega_{u}$ allows for adaptation of the differential equations to the spectrum requirements.

\subsection{Mixing technique}

By properly attenuating the eigenfunctions we obtain a set of legitimate waveforms. However, the utilization efficiency of the pulses might not be satisfactory. Here, we aim at enhancing the utilization efficiency by mixing various eigenfunctions.

Assume $\hat{y}_{n}(\omega)$ and $\hat{y}_{m}(\omega)$ are two scaled eigenfunctions that satisfy the mask requirement. It is not hard to check that all functions of the form $\mu \hat{y}_{n}(\omega)+\mathrm{j} \rho \hat{y}_{m}(\omega)$, where $\mu$ and $\rho$ are real constants, satisfy the same boundary conditions as $\hat{y}_{n}(\omega)$ and $\hat{y}_{m}(\omega)$. 
The benefit of mixing is that the modulus of the mixture depends on the modulus of both $\hat{y}_{n}$ and $\hat{y}_{m}$. We can take advantage of this fact by compensating the modulus of $\hat{y}_{n}$ with that of the $\hat{y}_{m}$ in regions where $\hat{y}_{n}$ lies far below the mask threshold, and vice versa. In other words, by properly mixing the two eigenfunctions, we can make the spectrum flatter in the constant regions of the mask, which ultimately increases the utilization efficiency.

To introduce the mixing technique more formally, let $\left\{\hat{y}_{n_{i}}\right\}_{i=1}^{2 N}$ be a subset of eigenfunctions for which the pairwise normalized inner products are small (in the sense of (13)). For arbitrary real numbers $\left\{\mu_{i}, \rho_{i}\right\}$ define

$\hat{Y}_{i}(\omega)=\left(\mu_{i} \hat{y}_{n_{2 i-1}}(|\omega|)+\mathrm{j} \rho_{i} \operatorname{sgn}(\omega) \hat{y}_{n_{2 i}}(|\omega|)\right) u\left(|\omega|-\omega_{l}\right)$,

where $\operatorname{sgn}(\cdot)$ and $u(\cdot)$ are the sign and Heaviside step functions, respectively. Due to the conjugate symmetry of $\left\{\hat{Y}_{i}(\omega)\right\}_{i=1}^{N}$, their representation $\left\{Y_{i}(t)\right\}_{i=1}^{N}$ in time are real-valued. Furthermore, they are almost orthogonal as follows:

$$
\begin{aligned}
\int_{-\infty}^{\infty} Y_{n}(t) Y_{m}(t)= & \int_{-\infty}^{\infty} \hat{Y}_{n}(\omega) \hat{Y}_{m}^{*}(\omega) \mathrm{d} \omega \\
= & \left.2 \mu_{n} \mu_{m} \int_{\omega_{l}}^{\infty} \hat{y}_{i_{2 n-1}}(\omega) \hat{y}_{i_{2 m-1}}(\omega)\right) \mathrm{d} \omega \\
& \left.+2 \rho_{n} \rho_{m} \int_{\omega_{l}}^{\infty} \hat{y}_{i_{2 n}}(\omega) \hat{y}_{i_{2 m}}(\omega)\right) \mathrm{d} \omega .
\end{aligned}
$$

Since the pairs $\left(\hat{y}_{i_{2 n-1}}, \hat{y}_{i_{2 m-1}}\right)$ and $\left(\hat{y}_{i_{2 n}}, \hat{y}_{i_{2 m}}\right)$ are almost orthogonal, the normalized inner products of $\left\{Y_{i}(t)\right\}_{i=1}^{N}$ can be made arbitrarily small. A similar argument is also valid for the decay rate and the finite energy of $\left\{\hat{Y}_{i}(\omega)\right\}_{i=1}^{N}$. In summary, by introducing the scalars $\left\{\mu_{i}, \rho_{i}\right\}$ as in (16), the already established properties of the waveforms stay valid while we can enhance the utilization efficiency.

\subsection{Pulses in the time-domain}

Up to this point, we have characterized the pulses in the Fourier domain by using differential equations. This is mainly motivated by the requirements imposed on the spectrum of the pulses. In practice, however, we need their representation in time domain. The interesting point which we will show is that the introduced pulses admit differential equations even in time domain. This property helps in implementing such pulses.

The following properties of Fourier transform are essential in our arguments:

$$
\begin{gathered}
\mathcal{F}_{\omega}^{-1}\left\{\omega^{m} \hat{f}(\omega)\right\}(t)=(2 \pi \mathrm{j})^{-m} f^{(m)}(t), \\
\mathcal{F}_{\omega}^{-1}\left\{\frac{\mathrm{d}^{m}}{\mathrm{~d} \omega^{m}} \hat{f}(\omega)\right\}(t)=\left(\frac{2 \pi t}{\mathrm{j}}\right)^{m} f(t),
\end{gathered}
$$

where $\mathcal{F}_{\omega}^{-1}\{\cdot\}(t)$ stands for the inverse Fourier operator with respect to $\omega$ at point $t$. For the eigenfunctions we have that

$$
\begin{gathered}
\mathcal{F}_{\omega}^{-1}\left\{\frac{\mathrm{d}}{\mathrm{d} \omega}\left[p(\omega) \dot{\hat{y}}_{n}(\omega)\right]\right\}(t)=\frac{2 \pi t}{\mathrm{j}} \sum_{i=0}^{k} a_{i} \mathcal{F}_{\omega}^{-1}\left\{\omega^{i} \dot{\hat{y}}_{n}(\omega)\right\}(t) \\
=-(2 \pi)^{2} t \sum_{i=0}^{k} \frac{a_{i}}{(2 \pi \mathrm{j})^{i}}\left(t y_{n}^{(i)}(t)+i y_{n}^{(i-1)}(t)\right) .
\end{gathered}
$$

Similarly,

$\mathcal{F}_{\omega}^{-1}\left\{\left(q(\omega)+\lambda_{n}\right) \hat{y}_{n}(\omega)\right\}(t)=\sum_{i=0}^{k-2} \frac{\tilde{b}_{i}}{(2 \pi \mathrm{j})^{i}} y_{n}^{(i)}(t)$,

where $\tilde{b}_{0}=b_{0}+\lambda_{n}$ and $\tilde{b}_{i}=b_{i}$ for $0<i \leq k-2$. The differential equation (2) in frequency domain translates into

$$
\begin{aligned}
& \sum_{i=0}^{k-2} \frac{(2 \pi)^{2} a_{i} t^{2}-2 \pi \mathrm{j}(i+1) a_{i+1} t-\tilde{b}_{i}}{(2 \pi \mathrm{j})^{i}} y_{n}^{(i)}(t) \\
& +\frac{(2 \pi)^{2} t^{2} a_{k-1}-2 \pi \mathrm{j} k a_{k} t}{(2 \pi \mathrm{j})^{k-1}} y_{n}^{(k-1)}(t)+\frac{2 \pi^{2} a_{k} t^{2}}{(2 \pi \mathrm{j})^{k}} y_{n}^{(k)}(t)=0
\end{aligned}
$$

in time domain. This shows a $k$ th order differential equation for $y_{n}(t)$. The simplest case which fits in conditions of Theorem 1 is $k=4$. For this case we have that

$$
\begin{gathered}
\tilde{a}_{4} y_{n}^{(4)}(t)+\frac{\mathrm{j} \tilde{a}_{3} t+4 \tilde{a}_{4}}{t} y_{n}^{(3)}(t)-\frac{\tilde{a}_{2} t^{2}-3 \mathrm{j} \tilde{a}_{3} t-\tilde{b}_{2}}{t^{2}} y_{n}^{(2)}(t) \\
-\frac{\mathrm{j} \tilde{a}_{1} t^{2}+2 \tilde{a}_{2} t-\mathrm{j} \tilde{b}_{1}}{t^{2}} y_{n}^{(1)}(t)+\frac{\tilde{a}_{0} t^{2}-\mathrm{j} \tilde{a}_{1} t-\tilde{b}_{0}}{t^{2}} y_{n}(t)=0
\end{gathered}
$$

where $\tilde{a}_{i}=\frac{a_{i}}{(2 \pi)^{i}}$ and $\tilde{b}_{i}=\frac{\bar{b}_{i}}{(2 \pi)^{i+2}}$. Since the function $y_{n}(t)$ is complex-valued, we can assume $y_{n}(t)=r_{n}(t)+\mathrm{j} q_{n}(t)$, where $r_{n}$, $q_{n}$ are real-valued functions. Fig. 2 depicts a block diagram for the circuit implementation of the functions $r_{n}, q_{n}$ for the case of $k=4$.

\section{Simulation results}

We study an example to show the efficiency of the proposed method. Let us consider the Sturm-Liouville boundary value problem formed by

$p(\omega)=\omega^{4}+\omega^{3}+10^{5}, q(\omega)=2.6 \omega^{2}, s(\omega)=1$.

This example is used as a proof of concept and does not reflect an optimized set of functions $p$ and $q$. However, one can check that, the functions in (23) satisfy the requirements of Theorem 1. We obtain the eigenfunctions by using a MATLAB toolbox called MATSLICE [17]. The toolbox provides us with the eigenvalues $\left\{\lambda_{n}\right\}_{n}$, eigenfunctions $\left\{\hat{y}_{n}(\omega)\right\}_{n}$ and their derivatives $\left\{\dot{\hat{y}}_{n}(\omega)\right\}_{n}$ between the boundary points. For a given $n$, by having $\lambda_{n}$ and $\left.\hat{y}_{n}(\omega)\right\}_{n}, \dot{\hat{y}}_{n}(\omega)$ at a particular $\omega$, we can convert the boundary value problem to an ODE, and extend the evaluation of $\hat{y}_{n}(\omega)$ to the whole real axis.

In this example, we evaluate the first 10 eigenfunctions on the interval $\frac{\omega}{2 \pi} \in[3.1 \mathrm{GHz}, 1 \mathrm{THz}]$. Next, we divide the eigenfunctions into pairs and use the mixing technique introduced in Section 4.3 to combine the two elements of each pair. The weights of the combinations are chosen optimally to maximize the spectral utilization efficiency of the final waveform over the range [3.1 GHz, 18 GHz]. We measure the utilization efficiency in terms of NESP [13]. As defined in (1), NESP is the ratio of the power transmitted in the designated passband over the total permissible power. An exhaustive search over all partitions of the 10 eigenfunctions into pairs reveals that, the best 4 waveforms are obtained by the pairs $(1,2),(3,6),(4,8)$, and $(5,10)$ (the fifth pair is ignored here). In addition to mixing, we attenuate the spectrum of the final pulse by a scalar in each of the intervals [3.1 GHz, $10.6 \mathrm{GHz}$ ] and $[10.6 \mathrm{GHz}, \infty)$ to make sure that they satisfy the FCC mask requirements.

In Figs. 3 and 4, we depict the spectrum of the obtained pulses. The shape of the pulses in time are also plotted in Figs. 5 and 6. If we base the spectral utilization efficiency of the pulses on the interval [3.1 GHz, $18 \mathrm{GHz}$ ], then, the NESP values would be $67.36 \%$, $64.16 \%, 63.75 \%$ and $63.63 \%$, respectively. However, based on the total available spectrum $[0 \mathrm{GHz}, 18 \mathrm{GHz}]$, the NESP values would drop to $58.87 \%, 56.07 \%, 55.71 \%$ and $55.61 \%$, respectively. This is due to the fact that the range $[0 \mathrm{GHz}, 3.1 \mathrm{GHz}]$ is discarded in designing the pulses.

The pairwise orthogonality is one of the requirements for the set of pulses. Although our results in this paper can guarantee only almost orthogonality of a properly selected set of pulses, we 


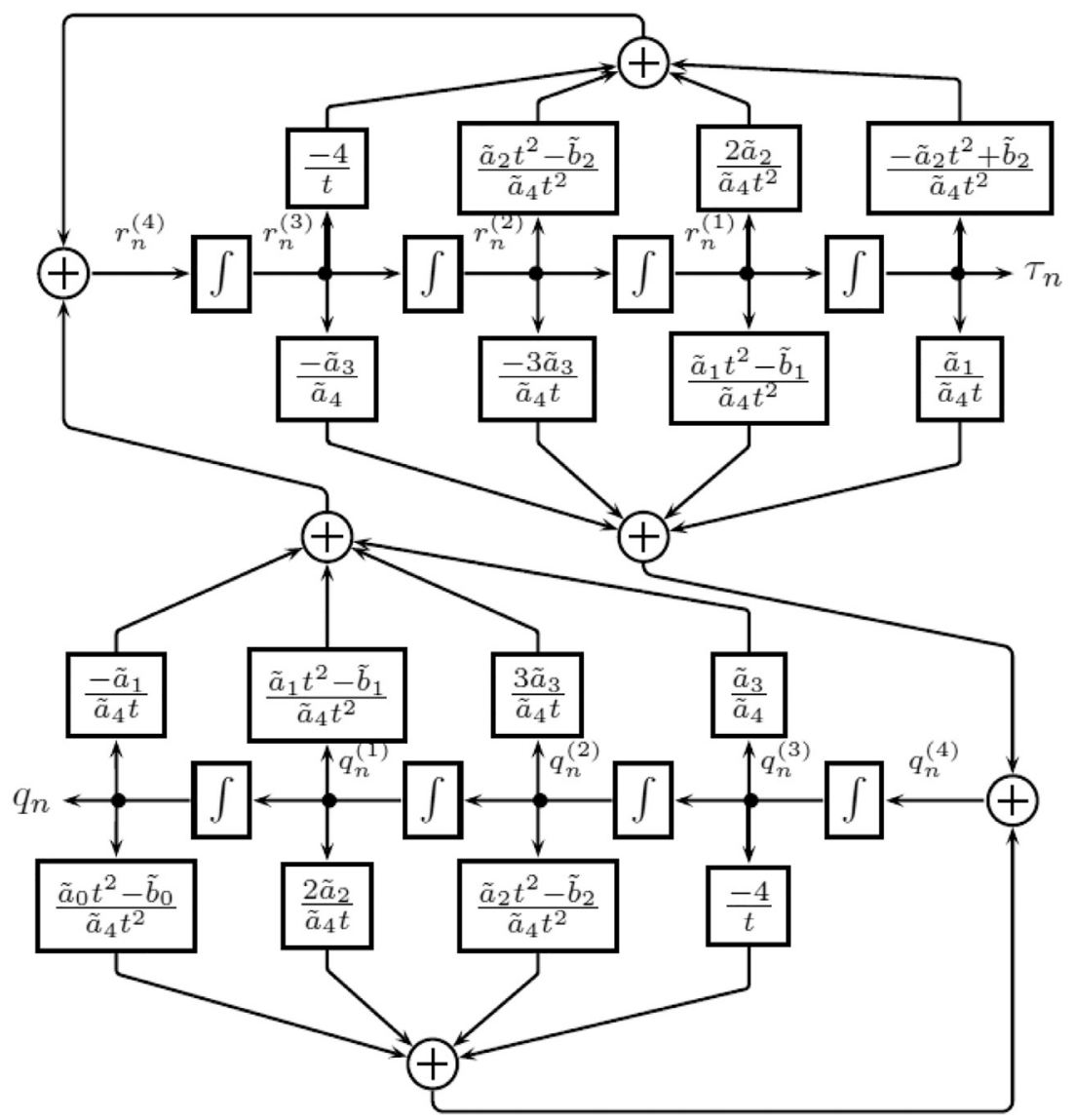

Fig. 2. The analog linear time-varying circuit producing designed pulses.

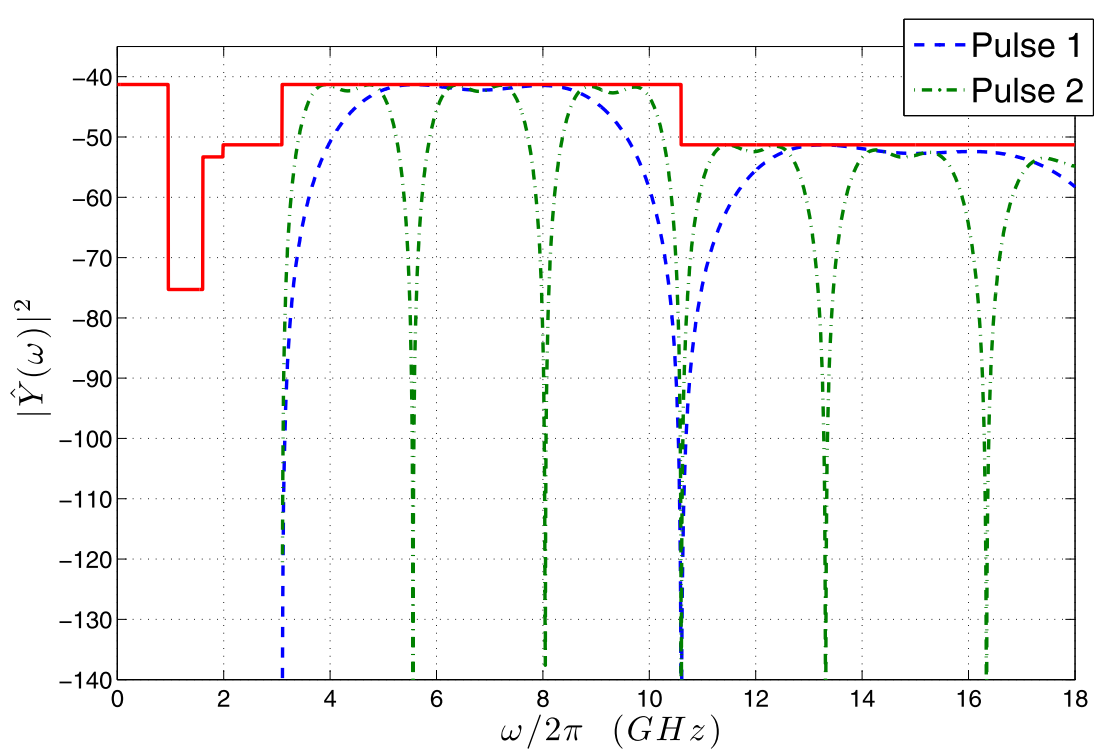

Fig. 3. FCC mask and the spectrum of the pulses obtained by optimally applying the mixing technique on eigenfunction pairs (1,2) as Pulse 1 and (3,6) as Pulse 2 .

observe that all the eigenfunctions of the Sturm-Liouville problem are orthogonal. To investigate this property for the generated pulses $\left\{y_{i}\right\}_{i=1}^{4}$ (after mixing), we consider the angles between the waveforms defined by

$\measuredangle_{i, j}=\cos ^{-1}\left(\frac{\int_{\mathbb{R}} y_{i}(t) y_{j}(t) \mathrm{d} t}{\left\|y_{i}\right\|_{2} \cdot\left\|y_{j}\right\|_{2}}\right)$ where $\|\cdot\|_{2}$ represents the $L_{2}$ norm of the function. Table 1 demonstrates the values of the pairwise angles very close to $90^{\circ}$, which reflects orthogonality. The small deviation from orthogonality is caused by truncation of the spectrum and numerical rounding involved in derivations.

The orthogonality of pulses is most effective in fully synchronized communications. In practice, however, exact synchronization is rarely achieved. Oftentimes, the data is extracted by de- 


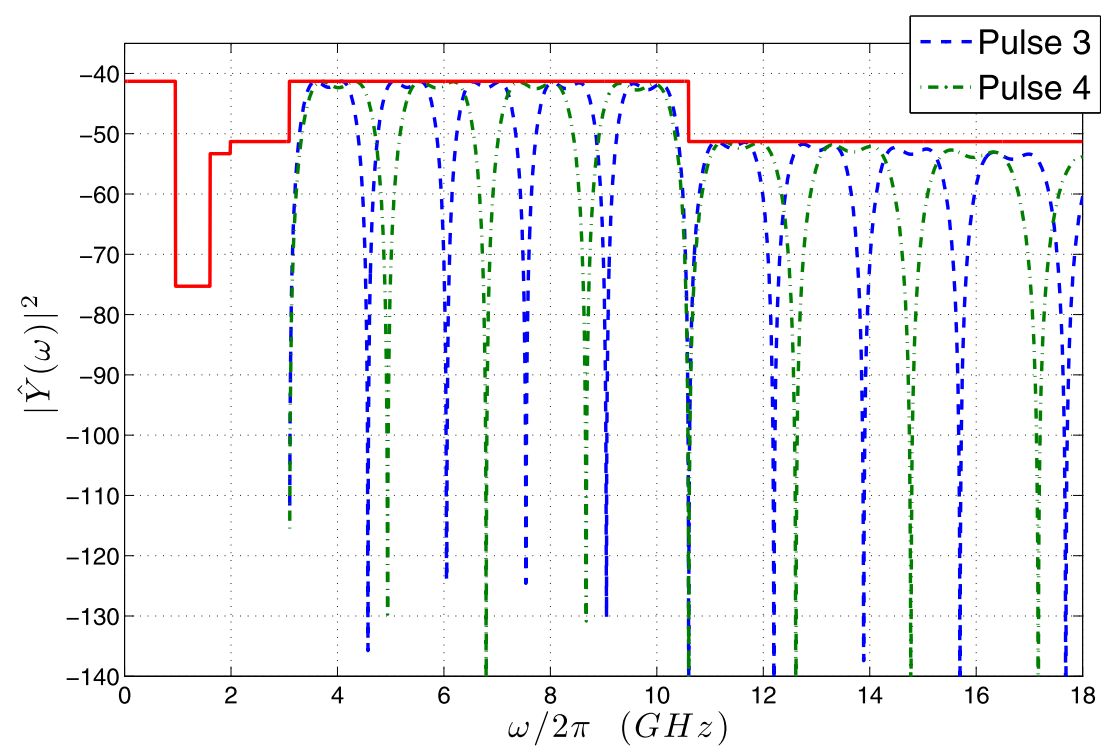

Fig. 4. FCC mask and the spectrum of the pulses obtained by optimally applying the mixing technique on eigenfunction pairs (4,8) as Pulse 3 and (5,10) as Pulse 4 .

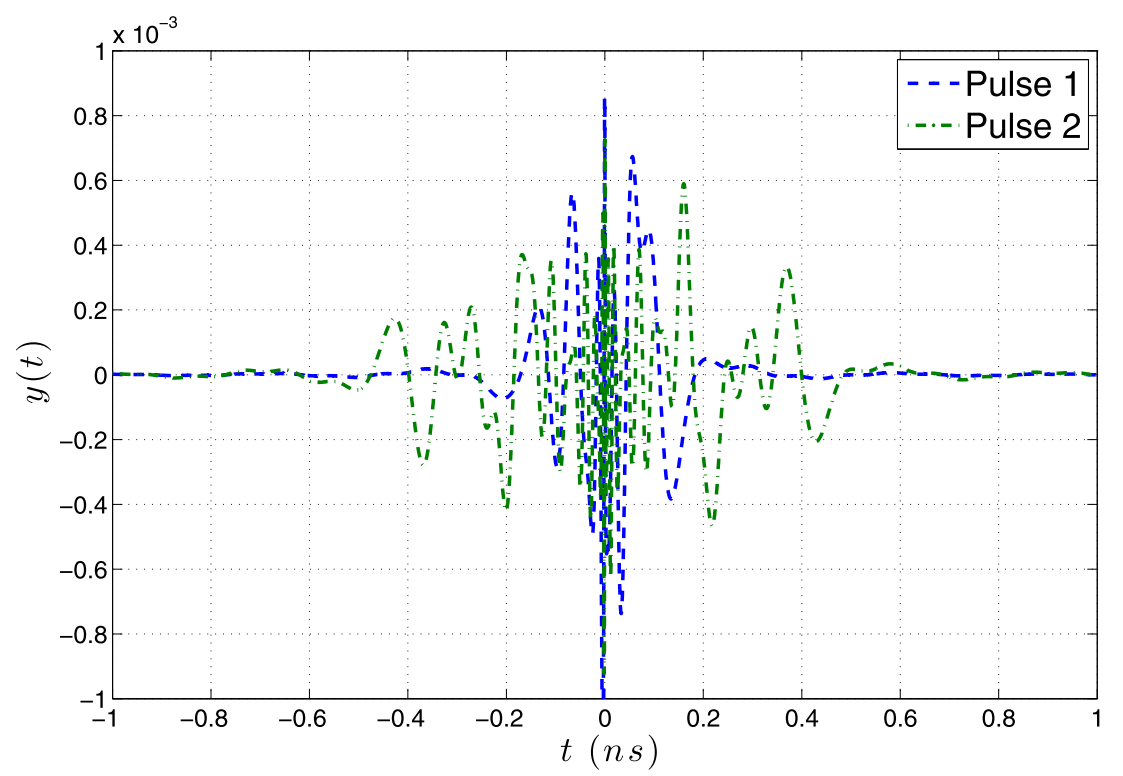

Fig. 5. Time-domain representation of pulses obtained by optimally applying the mixing technique on eigenfunction pairs (1,2) as Pulse 1 and (3,6) as Pulse 2 .

Table 1

The angles between the pulses.

\begin{tabular}{lllll}
\hline$\varkappa_{i, j}$ & $y_{1}$ & $y_{2}$ & $y_{3}$ & $y_{4}$ \\
\hline$y_{1}$ & $0^{\circ}$ & $90.64^{\circ}$ & $90.49^{\circ}$ & $90.39^{\circ}$ \\
$y_{2}$ & $90.64^{\circ}$ & $0^{\circ}$ & $90.36^{\circ}$ & $90.31^{\circ}$ \\
$y_{3}$ & $90.49^{\circ}$ & $90.36^{\circ}$ & $0^{\circ}$ & $90.27^{\circ}$ \\
$y_{4}$ & $90.39^{\circ}$ & $90.31^{\circ}$ & $90.27^{\circ}$ & $0^{\circ}$ \\
\hline
\end{tabular}

tecting the peaks of the correlation of the received signal with known pulses. Thus, it is desirable that the pulses have sharp autocorrelation peaks and the cross-correlations be relatively small to these peaks. In Figs. 7 and 8 we depict the correlation curves for $y_{1}$ and $y_{4}$, respectively.

\section{Comparison with other approaches}

There have been several optimal and sub-optimal waveform design methods for UWB communications in literature based on different approaches, such as multiband modulations, Gaussian derivatives, cognitive techniques, $\mathrm{QR}$ decomposition, differential equations and orthogonal functions. Here, a comparison between the method proposed in this paper and some other new techniques are presented.

In reference [18] and similar papers, a method for the design of nonlinear phase, full-band, UWB pulses that can satisfy a specified power spectrum mask has been introduced. These methods use convex programming in each step of the iterative procedure that updates the phase distribution of the goal function. These approaches are based on an optimization procedure that iteratively searches for the best solution and no direct solution to the problem is derived. Our method based on Sturm-Liouville boundary value problem, benefits from direct solution, less complexity and less computational cost.

In some other techniques, a particular mathematical functions such as Gaussian wave shape and its derivatives [19] or modified Hermite pulses [20] are used to design a set of optimal orthogonal waveforms. The waveform design is then converted into a 


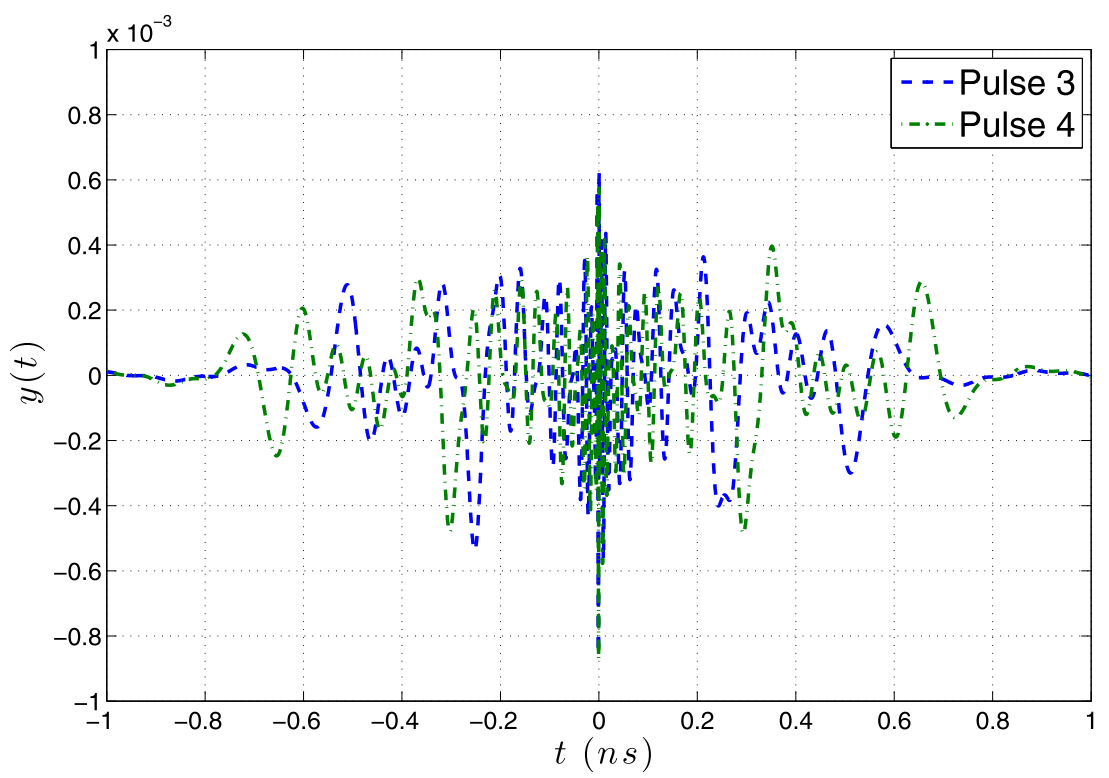

Fig. 6. Time-domain representation of pulses obtained by optimally applying the mixing technique on eigenfunction pairs (4,8) as Pulse 3 and $(5,10)$ as Pulse 4 .

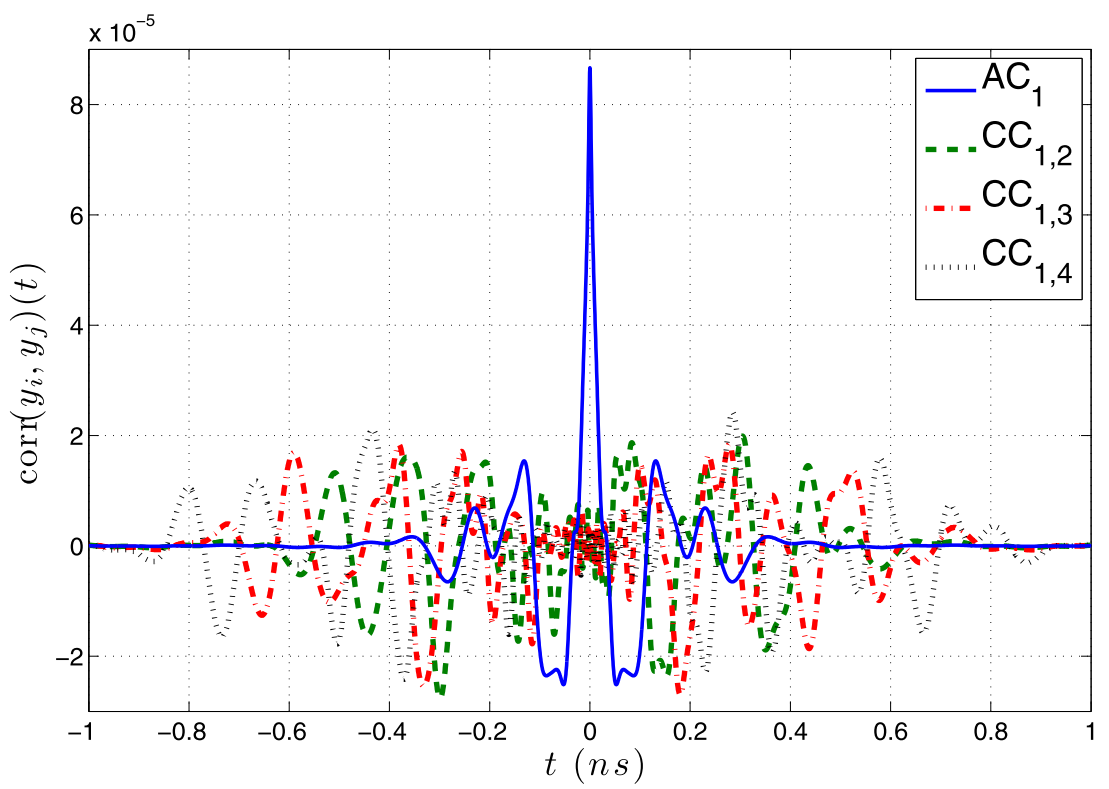

Fig. 7. The auto-correlation $(A C)$ and cross-correlation $(C C)$ of the pulse obtained from the eigenfunction pair $(1,2)$ with the rest of the pulses.

linear programming problem to be solved. Although the pulses can be designed to be orthogonal, however, this property is limited to the orthogonality of even and odd ordered pulses on each other. The orthogonality of our proposed pulses is an inherent property of the generated wave shapes and is followed from the mathematical solution of the governing differential equations.

Another popular pulse shaping technique uses cognitive methods with physical-layer quality of service constraints. These methods try to achieve an acceptable compromise between the spectral emission, data rate and synchronization errors robustness. As an example of such methods, in reference [21] a modified version of the Parks-McClellan technique has been proposed to fulfill these requirements. However, the rate and synchronization constraints, which bound the optimization space, cannot be easily handled and the resulting formulation cannot be reshaped as a convex or a linear programming. Moreover, the pulses generated lack the orthogonality properties which is a very important advantage of the proposed pulses using Sturm-Liouville boundary value problem. The piecewise spectral efficiency of this method is between $20 \%$ and $30 \%$. It also needs about 6 iterations and 32 quantization levels to generate the pulses.

In a different approach [14], pulses are built as linear combinations of known mathematical functions such as Hermite polynomials. Restrictions of orthogonality and spectral efficiency are then used to construct a search procedure to find the best set of waveforms. In contrast to our method, these techniques require comprehensive calculations and suffer from lower spectrum utilization efficiency between $20 \%$ and $30 \%$.

In a new method presented in $[22,23]$ UWB waveform division multiple access (WDMA) systems employ combinations of orthogonal wavelet pulses. Afterwards, the weighting coefficients are optimized by an iterative interference alignment approach based on maximum signal to interference plus noise ratio (Max-SINR) criterion. Compared to our approach, this method suffers from a low 


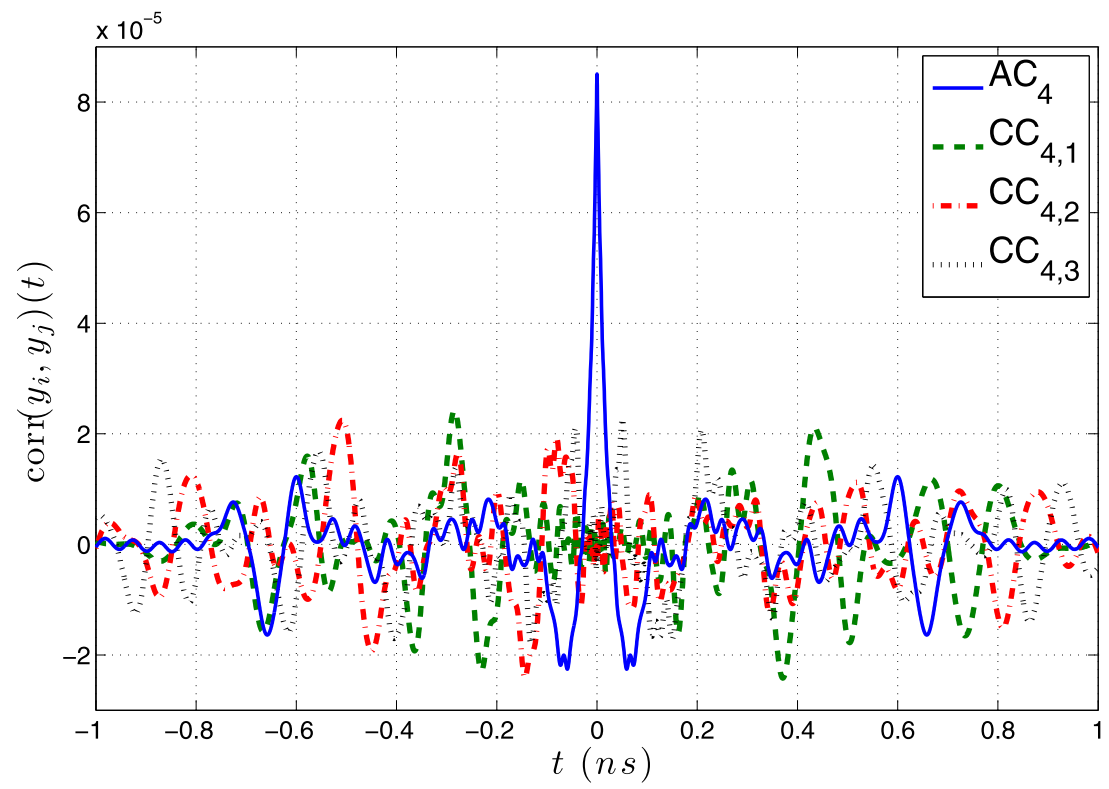

Fig. 8. The auto-correlation $(A C)$ and cross-correlation $(C C)$ of the pulse obtained from the eigenfunction pair $(5,10)$ with the rest of the pulses.

Table 2

Comparison between different approaches of orthogonal UWB pulse generation.

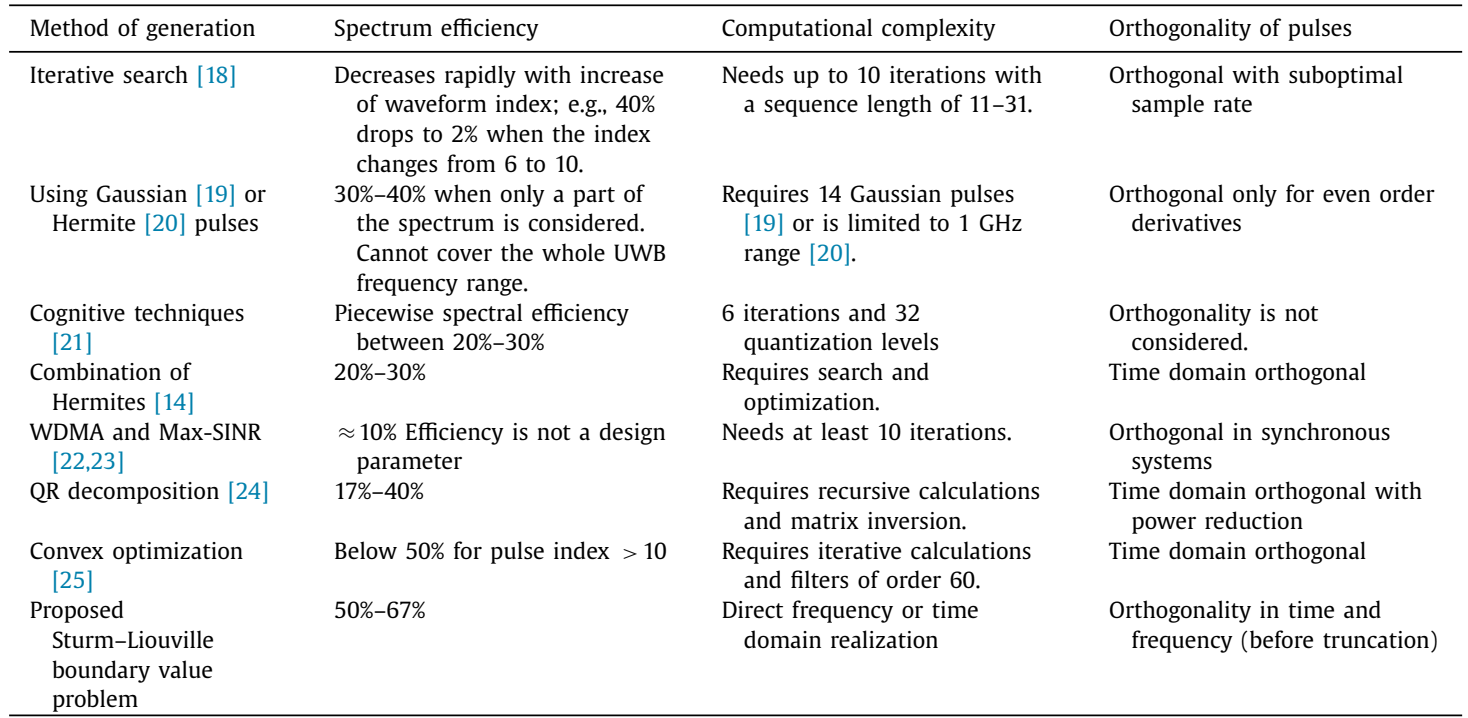

spectral efficiency of about $10 \%$ and the need for iterative calculations. Moreover, its orthogonality is limited to synchronous systems.

In [24] the design of multiple orthogonal wave shapes for impulse radio UWB (IR-UWB) has been investigated under the constraints of the FCC regulations. In this technique multiple orthogonal pulses are generated by applying the Gram-Schmidt polynomial and the orthogonalization via $\mathrm{QR}$ decomposition. The main drawback of this method is its low spectrum efficiency of below $40 \%$ and the need for higher order filters.

The proposed design in [25] is an extension of the iterative constrained elliptic error minimization for a single pulse to the case of multiple orthogonal pulses by incorporating the orthogonality conditions. Iterative calculations and higher pulse index are the main drawback of this method. Filters of order 60 are needed for the implementation of this method.
A comparison between the above mentioned methods and the proposed technique based on Sturm-Liouville boundary value problem is summarized in Table 2 .

\section{Conclusion}

In this paper, we investigated the design of orthogonal UWB pulses using Sturm-Liouville theory. The design is mainly in the frequency domain, however, due to the structure of the considered differential equations, the time domain implementation is realizable. The introduced waveforms have two tunable notch frequencies in their spectrum which allow us to adapt them to various requirements. In addition, they are pairwise orthogonal in two frequency intervals. It is also shown that the utilization efficiency of all the pulses are almost the same (55\%), which makes them fair choices for transmission pulses in multi-user systems. 


\section{Conflicts of interest statement}

The authors declare that they have no known competing financial interests or personal relationships that could have appeared to influence the work reported in this paper.

\section{Acknowledgment}

The authors would like to thank M. Abolhasani for her supports regarding the simulation results.

\section{Appendix A. Proof of Theorem 1}

Claim 1 is readily available by referring to the known results of Sturm-Liouville theory explained in Section 2.2. Since $s(\omega) \equiv 1$, there is no need for a weight functional and the eigenfunctions are orthogonal in the conventional sense.

The concept of Lyapunov stability is a common approach in analyzing the asymptotic behavior of the solutions of differential equations. It consists of forming a potential function in terms of a solution and its derivatives that asymptotically converges. The convergence of the potential function usually characterizes the asymptotic behavior of the solution. We will use the same technique to prove the claims of Theorem 1.

For introducing a suitable potential function, we consider the auxiliary function $z_{n}(\omega)=\hat{y}_{n}(\omega) \omega^{\frac{k-1}{2}}$ instead of $\hat{y}_{n}(\cdot)$. This is motivated by the fact that claim 2 of Theorem 1 is equivalent to $\lim _{\omega \rightarrow \infty} z_{n}(\omega)=0$. Our next step is to represent $z_{n}(\cdot)$ as a solution of a differential equation achieved by

$\left\{\begin{array}{l}\hat{y}_{n}(\omega)=\omega^{-\frac{k-1}{2}} z_{n}(\omega), \\ \dot{\hat{y}}_{n}(\omega)=\omega^{-\frac{k+1}{2}}\left(\frac{1-k}{2} z_{n}(\omega)+\omega \dot{z}_{n}(\omega)\right), \\ \ddot{\hat{y}}_{n}(\omega)=\omega^{-\frac{k+3}{2}}\left(\frac{k^{2}-1}{4} z_{n}(\omega)-(k-1) \omega \dot{z}_{n}(\omega)+\omega^{2} \ddot{z}_{n}(\omega)\right) .\end{array}\right.$

Thus, $z_{n}(\cdot)$ satisfies

$\ddot{z}_{n}(\omega)+\alpha(\omega) \dot{z}(\omega)+\beta_{n}(\omega) z_{n}(\omega)=0$,

where

$\left\{\begin{array}{l}\alpha(\omega)=\frac{\dot{p}(\omega)}{p(\omega)}-\frac{k-1}{\omega}=\frac{\sum_{i=0}^{k}(i+1-k) a_{i} \omega^{i}}{\sum_{i=0}^{k} a_{i} \omega^{i+1}}, \\ \beta_{n}(\omega)=\frac{\left(k^{2}-1\right) p(\omega)-2(k-1) \omega \dot{p}(\omega)+4 \omega^{2}\left(q(\omega)+\lambda_{n}\right)}{4 \omega^{2} p(\omega)} \\ =\frac{\gamma_{n}(\omega)+\sum_{i=2}^{k}\left(4 b_{i-2}-(k-1)(2 i-k-1) a_{i}\right) \omega^{i}}{4 \sum_{i=0}^{k} a_{i} \omega^{i+2}}, \\ \gamma_{n}(\omega)=\left(k^{2}-1\right) a_{0}+(k-1)^{2} a_{1} \omega+4 \lambda_{n} \omega^{2} .\end{array}\right.$

We need to study the asymptotics of $\alpha(\cdot)$ and $\beta(\cdot)$ which play significant roles in the asymptotics of $z_{n}(\cdot)$. Due to admitting a rational representation as in (A.3), the asymptotic behavior of each function is governed by the leading terms of the associated numerator and denominator. That means $\alpha(\omega) \approx \frac{1}{\omega}$ and $\beta(\omega) \approx \frac{\beta_{\infty}}{\omega^{2}}$, where $\approx$ stands for the asymptotic equivalence and

$\beta_{\infty}=\lim _{\omega \rightarrow \infty} \omega^{2} \beta_{n}(\omega)=\frac{1}{4}\left(\frac{b_{k-2}}{a_{k}}-\left(\frac{k-1}{2}\right)^{2}\right)>0$.

The positive sign of $\beta_{\infty}$ is a consequence of condition (9) in Theorem 1. An interesting observation is that, due to the condition $k \geq 4>2$, the asymptotics of $\alpha(\cdot)$ and $\beta(\cdot)$ are independent of $\lambda_{n}$ and therefore, $n$.

Next, we introduce the Lyapanov potential function as

$V_{n}(\omega)=\frac{\dot{z}_{n}^{2}(\omega)}{\beta_{n}(\omega)}+z_{n}^{2}(\omega)$.

Lemma 1. The function $V_{n}(\omega)$ converges to a finite value as $\omega \rightarrow \infty$.

The proof of Lemma 1 is provided in Appendix B. The key idea in the proof is that $V_{n}(\cdot)$ is positive and non-increasing for large enough values of $\omega$.
Although Lemma 1 can be used to obtain bounds on the decay rates of $z_{n}$ and $\dot{z}_{n}$, the results would depend on $\beta_{n}$ which has a rather complicated form. Instead, we use Lemma 2 proved in Appendix $C$ to simplify the analysis.

Lemma 2. The function

$\tilde{V}_{n}(\omega)=\omega^{2} \dot{z}_{n}^{2}(\omega)+\beta_{\infty} z_{n}^{2}(\omega)$

is convergent as $\omega \rightarrow \infty$.

Lemma 2 reveals that $z_{n}(\omega)$ and $\omega \dot{z}_{n}(\omega)$ are bounded for large values of $\omega$. Through (A.1) it is possible to interpret them as boundedness of $\omega^{\frac{k-1}{2}} \hat{y}_{n}(\omega)$ and $\omega^{\frac{k+1}{2}} \dot{\hat{y}}_{n}(\omega)$, which proves claim 2 of Theorem 1.

We conclude Claim 3 from Claim 2. The boundedness of $\omega^{\frac{k-1}{2}} \hat{y}_{n}(\omega)$ for $k \geq 4$ and the fact that $\hat{y}_{n}(\omega)$ is continuous, indicate that $\hat{y}_{n}(\omega)$ can be absolutely upperbounded by $M \times \max \left(1, \omega^{1.5}\right)$, where $M$ is a large enough scalar. The finite energy of the latter function is an upperbound for the energy of $\hat{y}_{n}$.

For the last claim we start by defining

$\theta_{n}(\omega)=\tan ^{-1} \frac{\omega \dot{z}_{n}(\omega)}{\sqrt{\beta_{\infty}} z_{n}(\omega)}$

which yields

$$
\begin{aligned}
\omega \dot{z}_{n}(\omega) & =\sqrt{\tilde{V}_{n}(\omega)} \sin \left(\theta_{n}(\omega)\right), \\
z_{n}(\omega) & =\sqrt{\frac{\tilde{V}_{n}(\omega)}{\beta_{\infty}}} \cos \left(\theta_{n}(\omega)\right) .
\end{aligned}
$$

Note that $\left\{\theta_{n}\right\}_{n}$ are functions while for Claim 4 we need scalars $\left\{\bar{\theta}_{n}\right\}_{n}$. We proceed by rewriting (5) as

$$
\begin{aligned}
\left(\lambda_{n}\right. & \left.-\lambda_{m}\right) \int_{\omega_{u}}^{\infty} \hat{y}_{n}(\omega) \hat{y}_{m}(\omega) \mathrm{d} \omega \\
& =\left.p(\omega)\left(\dot{\hat{y}}_{n}(\omega) \hat{y}_{m}(\omega)-\dot{\hat{y}}_{m}(\omega) \hat{y}_{n}(\omega)\right)\right|_{\infty} \\
& =\left.\frac{p(\omega)}{\omega^{k}}\left(\omega \dot{z}_{n}(\omega) z_{m}(\omega)-\omega \dot{z}_{m}(\omega) z_{n}(\omega)\right)\right|_{\infty} \\
& =\left.a_{k} \sqrt{\frac{\tilde{V}_{n}(\infty) \tilde{V}_{m}(\infty)}{\beta_{\infty}}} \sin \left(\theta_{n}(\omega)-\theta_{m}(\omega)\right)\right|_{\infty},
\end{aligned}
$$

where $\tilde{V}_{n}(\infty)$ represents $\lim _{\omega \rightarrow \infty} \tilde{V}_{n}(\omega)$. Eq. (A.9) relates the inner product of eigenfunctions to the asymptotic behavior of $\theta_{n} \mathrm{~s}$. Similar to the argument that $\hat{y}_{n} \mathrm{~s}$ have finite energy, we can use the decay bounds to show that their inner products are also finite. This implies that for all $n$ and $m$, we should have that

$\theta_{n, m}=\lim _{\omega \rightarrow \infty} \theta_{n}(\omega)-\theta_{m}(\omega)$,

where $\left\{\theta_{n, m}\right\}$ are scalars; i.e., the difference of $\theta_{n} \mathrm{~s}$ is asymptotically convergent. We define the claimed scalars $\left\{\bar{\theta}_{n}\right\}$ as

$\bar{\theta}_{n}=\theta_{n, 1}$.

It is now easy to rewrite (A.9) in the form of

$$
\begin{aligned}
& \left(\lambda_{n}-\lambda_{m}\right) \int_{\omega_{u}}^{\infty} \hat{y}_{n}(\omega) \hat{y}_{m}(\omega) \mathrm{d} \omega \\
& \quad=a_{k} \sqrt{\beta_{\infty}} \sqrt{\frac{\tilde{V}_{n}(\infty)}{\beta_{\infty}}} \sqrt{\frac{\tilde{V}_{m}(\infty)}{\beta_{\infty}}} \sin \left(\bar{\theta}_{n}-\bar{\theta}_{m}\right) .
\end{aligned}
$$

To complete the proof, we establish a connection between $M_{\hat{y}_{n}}$ and $\tilde{V}_{n}(\infty)$. By recalling the definition of $z_{n}$ we have

$$
\begin{aligned}
M_{\hat{y}_{n}} & =\limsup _{\omega \rightarrow \infty} \omega^{\frac{k-1}{2}} \hat{y}_{n}(\omega)=\limsup _{\omega \rightarrow \infty} z_{n}(\omega) \\
& =\sqrt{\frac{\tilde{V}_{n}(\infty)}{\beta_{\infty}}} \limsup _{\omega \rightarrow \infty} \cos \left(\theta_{n}(\omega)\right) .
\end{aligned}
$$


Our goal is to show that

$M_{\hat{y}_{n}}=\sqrt{\frac{\tilde{V}_{n}(\infty)}{\beta_{\infty}}}$

which justifies Claim 4. We consider two cases.

1. $\tilde{V}_{n}(\infty)=0$. This results in

$$
M_{\hat{y}_{n}}=\underbrace{\sqrt{\frac{\tilde{V}_{n}(\infty)}{\beta_{\infty}}}}_{0} \underbrace{\limsup _{\omega \rightarrow \infty} \cos \left(\theta_{n}(\omega)\right)}_{\leq 1}=0=\sqrt{\frac{\tilde{V}_{n}(\infty)}{\beta_{\infty}}} .
$$

2. $\tilde{V}_{n}(\infty)>0$. Let $\Theta_{n}=\limsup \sup _{\omega \rightarrow \infty} \cos \left(\theta_{n}(\omega)\right)$. In case of $\Theta_{n}=1$ we again obtain the desired result of (A.14). Hence, we focus on $\Theta_{n}<1$. In this case we have that $\Theta_{n}<\frac{1+\Theta_{n}}{2}<1$ and $0<\delta_{n}=$ $\frac{1-\Theta_{n}}{2\left(1+\Theta_{n}\right)}$. Thus, for large enough $\omega$ values we shall have that

$$
\begin{aligned}
& \left\{\begin{array}{l}
\tilde{V}_{n}(\omega) \leq\left(1+\delta_{n}\right) \tilde{V}_{n}(\infty) \\
\cos ^{2}\left(\theta_{n}(\omega)\right) \leq \frac{1+\Theta_{n}}{2}
\end{array}\right. \\
& \Rightarrow \beta_{\infty} z_{n}^{2}(\omega) \leq\left(\frac{3+\Theta_{n}}{4}\right) \tilde{V}_{n}(\infty) .
\end{aligned}
$$

By applying the definition of $\tilde{V}_{n}$ in (A.6), the above upperbound on $z_{n}^{2}(\omega)$ yields

$$
\begin{aligned}
\omega^{2} \dot{z}_{n}^{2}(\omega) & \geq \tilde{V}_{n}(\omega)-\left(\frac{3+\Theta_{n}}{4}\right) \tilde{V}_{n}(\infty) \\
& \geq\left(\frac{1-\Theta_{n}}{4}\right) \tilde{V}_{n}(\infty)=\kappa_{n}^{2},
\end{aligned}
$$

where $\kappa_{n}$ is strictly positive. For large $\omega \mathrm{s}$, this suggests that either $\dot{z}_{n}(\omega) \geq \frac{\kappa_{n}}{\omega}$, or $\dot{z}_{n}(\omega) \leq-\frac{\kappa_{n}}{\omega}$. In both cases $z_{n}$ cannot be bounded which is a contradiction. Therefore, $\Theta_{n}=1$ and (A.14) is valid.

\section{Appendix B. Proof of Lemma 1}

We showed in (A.4) that $\omega^{2} \beta_{n}(\omega)$ converges to a positive value. This confirms that $\beta_{n}(\omega)$ is positive for large enough values of $\omega$. Consequently, the potential function $V_{n}(\omega)$ defined in (A.5) is also non-negative for large values of $\omega$. We further compute that

$$
\begin{aligned}
\dot{V}_{n}(\omega) & =-\frac{\dot{\beta}_{n}(\omega)}{\beta_{n}^{2}(\omega)} \dot{z}_{n}^{2}(\omega)+\frac{2 \dot{z}_{n}(\omega)}{\beta_{n}(\omega)}(\underbrace{\ddot{z}_{n}(\omega)+\beta_{n}(\omega) z_{n}(\omega)}_{-\alpha(\omega) \dot{z}_{n}(\omega)}) \\
& =-(\underbrace{\frac{\dot{\beta}_{n}(\omega)}{\beta_{n}(\omega)}+2 \alpha(\omega)}_{v(\omega)}) \frac{\dot{z}_{n}^{2}(\omega)}{\beta_{n}(\omega)} .
\end{aligned}
$$

In Lemma 3 we prove that $v(\omega)$ is positive for large enough values of $\omega$. Hence, we conclude that $\dot{V}_{n}(\omega) \leq 0$; i.e., $V_{n}(\omega)$ is asymptotically both non-negative and non-increasing. This implies that $V_{n}(\omega)$ is convergent.

Lemma 3. For large enough values of $\omega$, we have that

$0<\frac{\dot{\beta}_{n}(\omega)}{\beta_{n}(\omega)}+2 \alpha(\omega)$

Proof. We show that the function

$\theta_{n}(\omega)=\frac{\beta_{n}(\omega) p^{2}(\omega)}{\omega^{2 k-2}}$ is asymptotically increasing. Recalling the definition of $\beta_{n}(\omega)$ from (A.3), we can rewrite $\theta_{n}(\omega)$ as

$$
\begin{aligned}
\theta_{n}(\omega)= & \frac{\left(k^{2}-1\right) p^{2}(\omega)-2(k-1) \dot{p}(\omega) p(\omega)}{4 \omega^{2 k}} \\
& +\frac{4\left(q+\lambda_{n}\right) p(\omega)}{4 \omega^{2 k-2}}=\sum_{i=0}^{2 k} \tau_{i} \omega^{-i}
\end{aligned}
$$

where the first two coefficients are given by

$$
\left\{\begin{array}{l}
\tau_{0}=a_{k}^{2}\left(\frac{b_{k-2}}{a_{k}}-\left(\frac{k-1}{2}\right)^{2}\right)>0, \\
\tau_{1}=a_{k} a_{k-1}\left(\frac{b_{k-2}}{a_{k}}+\frac{b_{k-3}}{a_{k-1}}-\frac{k^{2}-3 k+2}{2}\right)<0 .
\end{array}\right.
$$

The inequalities are due to the conditions (9) and (10). Since $\tau_{0}>0, \theta_{n}(\omega)$ converges to a positive value asymptotically, while $\tau_{1}<0$ indicates that the convergence is from below. In other words, $\theta_{n}(\omega)$ and similarly, $\ln \theta_{n}(\omega)$, are asymptotically strictly increasing. Thus, for large values of $\omega$ we have that

$$
\begin{aligned}
0<\frac{\partial}{\partial \omega} \ln \theta_{n}(\omega) & =\frac{\dot{\beta}_{n}(\omega)}{\beta_{n}(\omega)}+2\left(\frac{\dot{p}_{n}(\omega)}{p_{n}(\omega)}-\frac{k-1}{\omega}\right) \\
& =\frac{\dot{\beta}_{n}(\omega)}{\beta_{n}(\omega)}+2 \alpha(\omega),
\end{aligned}
$$

which proves the lemma.

\section{Appendix C. Proof of Lemma 2}

In fact, $\tilde{V}_{n}(\omega)$ is asymptotically similar to $V_{n}(\omega)$, though it is not necessarily non-increasing:

$\tilde{V}_{n}(\omega)=\beta_{\infty} V_{n}(\omega)+\left(1-\frac{\beta_{\infty}}{\omega^{2} \beta_{n}(\omega)}\right) \omega^{2} \beta_{n}(\omega) \frac{\dot{z}_{n}^{2}(\omega)}{\beta_{n}(\omega)}$.

According to Lemma $1, V_{n}(\omega)$ is convergent. Hence, recalling (A.5), we see that $\frac{\dot{z}_{n}^{2}(\omega)}{\beta_{n}(\omega)}$ is bounded as $\omega \rightarrow \infty$. Furthermore,

$\lim _{\omega \rightarrow \infty} \omega^{2} \beta_{n}(\omega)=\beta_{\infty}$,

$\lim _{\omega \rightarrow \infty} 1-\frac{\beta_{\infty}}{\omega^{2} \beta_{n}(\omega)}=1-\frac{\beta_{\infty}}{\beta_{\infty}}=0$.

In summary, we conclude that

$\lim _{\omega \rightarrow \infty} \underbrace{\left(1-\frac{\beta_{\infty}}{\omega^{2} \beta_{n}(\omega)}\right)}_{\rightarrow 0} \underbrace{\omega^{2} \beta_{n}(\omega)}_{\rightarrow \beta_{\infty}} \underbrace{\frac{\dot{z}_{n}^{2}(\omega)}{\beta_{n}(\omega)}}_{\text {bounded }}=0$,

$\lim _{\omega \rightarrow \infty} \tilde{V}_{n}(\omega)=\beta_{\infty} \lim _{\omega \rightarrow \infty} V_{n}(\omega)<\infty$

\section{References}

[1] J. Taylor, Introduction to Ultra-Wideband Radar Systems, Boca Raton, FL: CRC, 1995.

[2] M. Ghavami, L.B. Michael, R. Kohno, Ultra Wideband Signals and Systems in Communication Engineering, 2 edition, John Wiley, 2007.

[3] S. Stroh, Ultra-wideband: multimedia unplugged, IEEE Spectr. 40 (9) (2003) 23-27.

[4] N. El-Fishawy, M. Shokair, W. Saad, Proposed MAC protocol versus IEEE 802.15.3a for multimedia transmission over UWB networks, Prog. Electromagn. Res. B 2 (2008) 189-206.

[5] N. Riaz, M. Ghavami, An energy-efficient adaptive transmission protocol for ultra-wideband wireless sensor networks, IEEE Trans. Veh. Technol. 58 (7) (2009) 3647-3660.

[6] R. Brown, N. Ghavami, H. Siddiqui, M. Adjrad, M. Ghavami, S. Dudley, Occupancy based household energy disaggregation using ultra wideband radar and electrical signature profiles, Elsevier Energy Build. 141 (2017) 134-141.

[7] B. Allen, M. Ghavami, A. Armogida, H. Aghvami, The holy grail of wire replacement? IEE Commun. Eng. Mag. 1 (5) (2003) 14-17. 
[8] W.T. Ang, C. Jie, L. Tiejun, High-order monocycle design and its waveformgenerating circuit for UWB communications, IEEE Trans. Circuits Syst. I 54 (8) (2007) 1657-1665.

[9] Q. Wang, J. Yao, UWB doublet generation using nonlinearly-biased electro-optic intensity modulator, Electron. Lett. 42 (22) (2006) 1304-1305.

[10] M. Ghavami, L.B. Michael, S. Haruyama, R. Kohno, A novel UWB pulse shape modulation system, Kluwer Wireless Personal Commun. J. 23 (2002) 105-120.

[11] R. Dilmaghani, M. Ghavami, B. Allen, H. Aghvami, Novel pulse shaping using prolate spheroidal wave functions for UWB, in: IEEE Intern. Symp. Personal, Indoor And Mobile Radio Comm. (PIMRC), Beijing, China, 1, 2003, pp. 602-606.

[12] F. C. Commission:, Revision of part 15 of the commissions rule regarding ultra-wideband transmission systems, First Report and Order, ET Docket 98-153, FCC 02-48, 2002.

[13] X. Wu, Z. Tian, T.N. Davidson, G.B. Giannakis, Optimal waveform design for UWB radios, IEEE Trans. Signal Proc. 54 (6) (2006) 2009-2021.

[14] J.A.N. da Silva, M.L.R. de Campos, Spectrally efficient UWB pulse shaping with application in orthogonal PSM, IEEE Trans. Commun. 55 (2) (2007) 313-322.

[15] M. Ghavami, A. Amini, F. Marvasti, Unified structure of basic UWB waveforms, IEEE Trans. Circuits Syst. II 55 (12) (2008) 1304-1308.

[16] A. Zettl, Sturm-Liouville Theory, New York: Amer. Math. Soc., 2005.

[17] V. Ledoux, M.V. Daele, G.V. Berghe, MATSLISE: a MATLAB package for the numerical solution of Sturm-Liouville and Schrödinger equations, ACM Trans. Math. Softw. 31 (4) (2005) 532-554.
[18] I. Dotlic, R. Kohno, Design of the family of orthogonal and spectrally efficient UWB waveforms, IEEE J. Sel. Top. Signal Process. 1 (4) (2007) 21-30.

[19] Y. Guo, Optimal waveform design for ultra-wideband communication based on gaussian derivatives, IEEE J. Commun. Netw. 10 (4) (2008) 451-454.

[20] Y.G. Li, M.R. Haider, Y. Massoud, An efficient orthogonal pulse set generator for high-speed sub-ghz UWB communications, IEEE International Symposium on Circuits and Systems (ISCAS), Melbourne, Australia, 2014.

[21] M. Biagi, V. Polli, Ultrawide band cognitive pulse shaping under physical layer QoS constraints, IEEE Trans. Commun. 59 (11) (2011) 3167-3176.

[22] Z. Yin, S. Wu, Z Shi, Z Wu, A new design of pulse waveform for waveform division multiple access UWB wireless communication system, in: IEEE International Conference on Communication Software and Networks, Beijing, China, 2016.

[23] Z. Yin, M. Wu, S. Wu, Z. Wu, Y. Chen, IA-OPD: An optimized orthogonal pulse design scheme for waveform division multiple access UWB systems, IEEE Syst. J. 13 (1) (2019) 1-10.

[24] S. Kim, Y. Kim, X. Li, J. Kang, Orthogonal pulse design in consideration of FCC and IEEE 802.15.4a constraints, IEEE Commun. Lett. 17 (5) (2013) 896-899.

[25] N. Jiangli, W. Qiong, L. Xiaoping, Design of multiple orthogonal pulses for UWB radios using an iterative convex constrained $l_{1}$ minimization method, in: 29th Chinese Control And Decision Conference (CCDC), Chongqing, China, 2017. 